\title{
¿Cómo registramos en las historias clínicas de nuestros pacientes que hay un niño o adolescente con discapacidad en la familia? Estudio de corte transversal
}

How do we record in our patients' medical records the presence of a child or adolescent with a disability in the family? A cross-sectional study

Micaela Alurralde ${ }^{a}$, Paula Carrete ${ }^{a}$, Celeste Puga ${ }^{b, c}$, Sergio Terrasa ${ }^{b, a, d}$

\begin{abstract}
Resumen
Introducción. Para cuidar a las familias de una persona con discapacidad es necesario correr el foco desde el paciente individual hacia su sistema familiar, tarea difícil de implementar en el ámbito de la atención primaria, y para la que no se cuenta con datos locales respecto de esta problemática.

Objetivo. Describir qué proporción de las historias clínicas de las personas adultas que integran una familia en la que hay un niño o adolescente menor de 18 años con discapacidad tiene documentada esta información contextual.

Métodos. Fueron revisadas hasta el 31/12/19, 250 historias clínicas electrónicas de adultos familiares de primer grado de una muestra aleatoria de 153 menores de 18 años de edad afiliados al Plan de Salud del Hospital Italiano de Buenos Aires que tenían Certificado Único de Discapacidad.

Resultados. Estos 250 adultos habían realizado una mediana de seis consultas con su médico de cabecera. Solamente en 117 (47\%, intervalo de confianza del $95 \% 40$ a 53) historias clínicas electrónicas estaba documentado que en su familia directa había un menor con discapacidad, y sólo en 18 (7\%) esta información estaba consignada en su lista de problemas.

Conclusiones. En más de la mitad de la muestra de adultos estudiada no está documentado en su historia clínica electrónica que en la familia de esa persona hay un menor con discapacidad. Estos hallazgos llaman la atención, por tratarse de un sistema de salud que ofrece atención coordinada a través de la figura de un médico de cabecera.
\end{abstract}

\begin{abstract}
Introduction. In order to care for the families of a person with a disability, it is necessary to shift the focus from the individual patient to their family system. This is a difficult task to implement in the primary care field and for which no local data on this issue are available.

Objective. To describe the proportion of electronic medical records of adult people in a family with a disabled child or adolescent under 18 years old in which this contextual information is documented.

Methods. A total of 250 electronic medical records were reviewed up to 31/12/19 regarding first-degree adult relatives from a random sample of 153 children under the age of 18 affiliated to the Health Maintenance Organization of the Hospital Italiano de Buenos Aires who had a Disability Certificate.

Results. These 250 adults had made an average of six consultations with their primary care physician. Only $117(47 \%$, $95 \%$ confidence interval 40 a 53) electronic medical records documented that their immediate family included a child with a disability, and only $18(7 \%)$ had this information on their problem list.

Conclusions. More than half of the sample of adults studied did not have documented in their electronic medical records that there was a child with a disability in their family. These findings are striking given that this Health Maintenance Organization offers coordinated care through the figure of a primary care physician.
\end{abstract}

Palabras clave: Niños con Discapacidad, Registros Electrónicos de Salud, Calidad de la Atención de Salud, Medicina Familiar y Comunitaria. Keywords: Disabled Children, Electronic Health Records, Quality of Health Care, Family Practice.

Alurralde M, Carrete P, Puga C, Terrasa S. ¿Cómo registramos en las historias clínicas de nuestros pacientes que hay un niño o adolescente con discapacidad en la familia? Estudio de corte transversal . Evid Actual Pract Ambul. 2021;24(4):e002157. Available from: https://dx.doi.org/10.51987/ EVIDENCIA.V25I1.6992.

\section{Introducción}

Como sostiene la Organización Mundial de la Salud (2001), se entiende por discapacidad a "...toda limitación en la actividad y/o restricción en la participación, originada en la interacción entre la persona con una condición de salud y los factores contextuales (entorno físico, humano, actitudinal y sociopolítico), para desenvolverse en su vida cotidiana, dentro de su entorno físico y social, según su sexo y edad..." ${ }^{1}$. Como afirma esta definición y la conceptualización aportada por el modelo social de la discapacidad, esta puede concebirse de un modo dinámico e integral, como un complejo proceso de salud, económico, sociocultural y político, en el que la discapacidad se construye a partir de la interacción entre las capacidades funcionales de la persona y su entorno físico y social $^{2}$.

\footnotetext{
a Servicio de Medicina Familiar y Comunitaria, Hospital Italiano de Buenos Aires. micaela.alurralde@hospitalitaliano.org.ar, paula.carrete@hospitalitaliano.org.ar

b Departamento de Investigación, Hospital Italiano de Buenos Aires.

c Gerencia Médica, Plan de Salud del Hospital Italiano. celeste.puga@hospitalitaliano.org.ar

d Departamento de Medicina, Instituto Universitario Hospital Italiano de Buenos Aires. sergio.terrasa@hospitalitaliano.org.ar
} 
El alivio de la persona con discapacidad depende de cualquier actor social que pueda aportar herramientas para mejorar su funcionalidad $^{2}$, siendo la familia la institución más accesible para producir ese alivio y para llevar adelante intervenciones preventivas y terapéuticas. Además, la familia se ve afectada en forma directa por la condición de discapacidad de cualquiera de sus miembros, y dado que el último Censo Nacional informó que en uno de cada cuatro hogares de Argentina hay al menos una persona con dificultades ${ }^{3}$, es fundamental que el equipo de salud preste atención al sistema familiar, no solamente para contener, paliar o acompañar el impacto que provoca la presencia de un miembro con discapacidad en su seno, sino también para mejorar la calidad de vida de todos sus miembros ${ }^{4}$.

Es sabido que los familiares o cuidadores principales de las personas con discapacidad asumen este rol por múltiples causas: por fines altruistas, por gratitud o por obligación, también para evitar la censura de familiares y amigos o sencillamente, porque son los únicos que pueden hacerlo ${ }^{5}$. Esta situación genera que, tanto la persona afectada por la discapacidad, como también su principal cuidador/a, experimenten cambios importantes en el desempeño de sus roles habituales ${ }^{6}$. Si bien el nivel de compromiso no es igual en todas las familias afectadas, las que cuentan con un miembro con discapacidad constituyen un grupo en riesgo de desarrollar problemas psicosociales, dependiendo el desarrollo y la manifestación de estos conflictos de la capacidad de adaptación de cada familia ${ }^{7}$.

Dada esta distribución de roles, es difícil conocer hasta qué punto están satisfechas las necesidades de salud del familiar que más se carga al hombro los cuidados de la persona con discapacidad, ya sea porque las desconoce o bien porque deja de lado su autocuidado en pos de enfocar todos sus esfuerzos en cuidar a quien considera el miembro más vulnerable de la familia. Esta situación se torna aún más complicada si se tiene en cuenta que, en general, los servicios de salud y los programas de rehabilitación centran la atención en la persona con discapacidad y olvidan las necesidades familiares y las de su cuidador/a principal ${ }^{5}$.

Por estas razones, para que el equipo de salud contribuya a mantener una buena calidad de vida familiar, es necesario correr el foco desde la persona afectada hacia el de la familia afectada. Es recomendable que el equipo pesquise qué rol ocupa cada participante de la familia, el estado de las relaciones y las interacciones familiares, cómo se llevan a cabo las actividades diarias, cuál es el ingreso económico familiar, si este puede mantenerse en el tiempo, etc. Esta información nos ayuda a establecer objetivos y a otorgar herramientas a las familias para la optimización del funcionamiento del equipo de trabajo alrededor de la persona con discapacidad, sin descuidar a ninguno del resto de sus miembros ${ }^{4}$.

Sin embargo, hasta la realización de esta investigación no teníamos documentada en forma sistemática la real magnitud de cuán al tanto estaban los médicos de cabecera de la presencia de un integrante con discapacidad en las familias bajo su cuidado. Es por eso que decidimos evaluar, en el sistema de salud en el que estamos inmersos, cuánto y cómo registran nuestros equipos de salud en la historia clínica de sus pacientes, la discapacidad de un familiar como una problemática que refleja su contexto vital.

\section{Objetivo}

Describir qué proporción de las historias clínicas de las personas que integran una familia en la que hay un niño o adolescente menor de 18 años con discapacidad tiene documentada esta información contextual.

\section{Métodos}

\section{Diseño y población}

Estudio de corte transversal llevado a cabo en el Hospital Italiano de Buenos Aires (HIBA), un hospital universitario que cuenta con una historia clínica electrónica (HCE) donde se registra toda la información médica del paciente, tanto clínica, como de prescripción de fármacos, procedimientos y estudios diagnósticos. Esta institución es el principal prestador de un seguro prepago de salud (denominado Plan de Salud del Hospital Italiano) que brinda servicios de salud a 164.034 afiliados (22\% de ellos, menores de 18 años).

Fueron consideradas elegibles para participar de esta investigación las familias que contaran con al menos un niño con discapacidad documentada y un adulto, ambos afiliados al seguro de salud mencionado. Fueron definidos como pacientes índice a aquellas personas menores de 18 años de edad que contaban con Certificado de Discapacidad emitido por la autoridad sanitaria competente. Para cada paciente índice, fueron elegibles sus familiares de primer grado (madre, padre, hermanos).

A partir de estos criterios, fue solicitado al Plan de Salud del Hospital Italiano de Buenos Aires el listado de afiliados menores de 18 años que contaban con Certificado de Discapacidad, a partir del cual fue seleccionada una muestra aleatoria de 200 sujetos. Posteriormente fue solicitada la lista de todos los afiliados que integraban el grupo familiar -registrados al momento de su afiliación a este seguro de salud- de los pacientes índice.

La investigadora principal (MA) revisó las HCE de los familiares adultos de primer grado de los pacientes índice, desde su fecha de nacimiento y hasta el 31 de diciembre de 2019. Fueron excluidos de la muestra final quienes no cumplían con los criterios de inclusión.

\section{Tamaño muestral}

Para el cálculo del tamaño muestral se asumió que solo el $20 \%$ de las HCE relevadas de los adultos responsables de la crianza del niño y/o adolescente índice con discapacidad tendría consignada información sobre este aspecto del contexto familiar. Procurando estimar esta prevalencia con un intervalo de confianza del $95 \%$ y una hemiamplitud de $7,5 \%$, habíamos estimado que sería necesario contar con un mínimo de 140 pacientes índice.

\section{Recolección de datos}

Durante la revisión de la HCE del familiar de cada paciente índica fue verificado en la lista de problemas y en los textos libres del registro de los episodios de cuidado (denominados evoluciones) si estaba documentado que ese adulto tenía relación de parentesco con el paciente con discapacidad.

Además, fue recolectada la información demográfica necesaria para describir la muestra y para construir el índice UPC de continuidad de los cuidados. El índice UPC (del inglés Usual provider of care) sintetiza el número de consultas realizadas al prestador habitual de cuidados de salud en un periodo de tiempo dado, en relación al número total de consultas realizadas a prestadores similares. Puede tomar valores entre 0 y 1 . Se calcula mediante la fórmula $U P C=n_{i} / N$; donde $n$ representa el número de consultas realizadas al prestador habitual en un periodo definido y $N$, al total de las visitas efectuadas durante el mismo período. En este trabajo fue considerado prestador habitual al médico de cabecera más consultado por el paciente durante el período revisado en la historia clínica electrónica.

\section{Análisis estadístico}

Las variables continuas se describieron calculando la media y desvío estándar o la mediana y el intervalo intercuartilo (IIC; percentilo 25 a 75), según su distribución; mientras que las variables 
categóricas se reportaron como proporciones. Para el desenlace principal (proporción de HCE con documentación de alguna información sobre el niño o adolescente con discapacidad) se reporta el intervalo de confianza (IC) del $95 \%$. Para el análisis de los datos se utilizó Stata (versión 13.0).

\section{Consideraciones éticas}

El protocolo de investigación fue aprobado por el Comité de Ética de Protocolos de Investigación del HIBA.

\section{Resultados}

A partir de una lista de 726 menores con discapacidad documentada fue obtenida de forma aleatoria una muestra de 200 pacientes. Tras la exclusión de los familiares que no cumplían los criterios de elegibilidad, finalmente fueron incluidos un total de 250 familiares adultos de primer grado de 153 menores de 18 años con discapacidad (ver Figura 1).

Figura 1. Diagrama que representa el proceso de identificación de los 250 adultos familiares de primer grado de pacientes menores de 18 años con discapacidad documentada por la autoridad sanitaria, afiliados al seguro de salud de un Hospital Universitario Privado del Área Metropolitana de Buenos Aires.

\begin{tabular}{|c|c|}
\hline $\begin{array}{l}\text { Base de datos: } 726 \text { pacientes } \\
\text { con discapacidad (menores } \\
\text { de } 18 \text { años) }\end{array}$ & \\
\hline$\downarrow$ & \\
\hline $\begin{array}{l}\text { Muestra aleatoria: } 200 \\
\text { pacientes con discapacidad }\end{array}$ & \\
\hline$\nabla$ & \multirow{4}{*}{$\begin{array}{l}431 \text { familiares excluidos por: } \\
\text { - ser menores de } 18 \text { años } \\
\text { - no encontrar evoluciones de } \\
\text { actividad en los } 2 \text { años } \\
\text { previos } \\
\text { - no poder determinar vínculo } \\
\text { con el paciente índice } \\
\text { - no poder identificar médico } \\
\text { de cabecera }\end{array}$} \\
\hline $\begin{array}{l}681 \text { pacientes obtenidos del } \\
\text { grupo familiar }\end{array}$ & \\
\hline 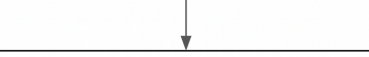 & \\
\hline $\begin{array}{l}250 \text { pacientes mayores de } 18 \\
\text { años (representan } 153 \\
\text { pacientes con discapacidad) }\end{array}$ & \\
\hline
\end{tabular}

Las principales características de los participantes con discapacidad se describen en la Tabla 1 y la de sus familiares adultos de primer grado, en la Tabla 2.

Como podemos observar en laTabla 3, solo en el $47 \%$ (IC $95 \%$ 40 a 53) de las historias clínicas de esta muestra de adultos que habían realizado una mediana de seis consultas con su médico de cabecera estaba documentado que en su familia directa había un menor con discapacidad, figurando esta información en su lista de problemas sólo en el $7 \%$. Asimismo, sólo el $57,2 \%$ de los familiares de primer grado contaban con información acerca de su contexto global (ocupación, grupo familiar, convivientes, etc).

Tabla 1. Características principales de una muestra de 153 pacientes menores de 18 años con discapacidad documentada por la autoridad sanitaria, afiliados al seguro de salud de un Hospital Universitario Privado del Área Metropolitana de Buenos Aires. Notas: ICC: intervalo intercuartilo (Percentilo 25 a 75). ${ }^{a}$ La suma de los porcentajes de los diferentes subtipos de discapacidad supera $100 \%$ ya que había pacientes con más de un subtipo de discapacidad.

\begin{tabular}{|c|c|c|}
\hline \multicolumn{2}{|l|}{ Características } & $\mathrm{N}=153$ \\
\hline \multicolumn{2}{|l|}{ Edad (años), mediana (IIC) } & $11(4$ a 18$)$ \\
\hline \multirow{5}{*}{$\begin{array}{l}\text { Tipo de discapacidad }{ }^{a}, \mathrm{n} \\
(\%)\end{array}$} & Mental & $123(80,4)$ \\
\hline & Motora & $15(9,8)$ \\
\hline & Sensorial & $15(9,8)$ \\
\hline & Visceral & $7(4,6)$ \\
\hline & Mixta & $6(3,9)$ \\
\hline
\end{tabular}

Tabla 2. Características de los 250 adultos familiares de primer grado de los 153 pacientes con discapacidad incluidos en el estudio, afiliados al seguro de salud de un Hospital Universitario Privado del Área Metropolitana de Buenos Aires. Nota: ICC: intervalo intercuartilo (Percentilo 25 a 75).

\begin{tabular}{|l|l|c|}
\hline \multicolumn{2}{|l|}{ Características } & $\mathrm{N}=250$ \\
\hline \multicolumn{2}{|l|}{ Edad (años), mediana (IIC) } & $\begin{array}{c}44(39 \mathrm{a} \\
48)\end{array}$ \\
\hline \multicolumn{1}{|l|}{ Sexo femenino, $\mathrm{n}(\%)$} & $154(62)$ \\
\hline Parentesco, $\mathrm{n}(\%)$ & Madre & $138(55,4)$ \\
\cline { 2 - 3 } & Padre & $86(34,5)$ \\
\cline { 2 - 3 } & Hermana & $16(6,4)$ \\
\cline { 2 - 3 } & Hermano & $9(3,6)$ \\
\hline Ocupación, n (\%) & Profesional independiente & $35(14)$ \\
\cline { 2 - 3 } & Empleado/a administrativo/a & $29(11,6)$ \\
\cline { 2 - 3 } & Encargado/a del hogar & $28(11,2)$ \\
\cline { 2 - 3 } & Comerciante & $21(8,4)$ \\
\cline { 2 - 3 } & Estudiante & $16(6,4)$ \\
\cline { 2 - 3 } & Docente & $13(5,2)$ \\
\cline { 2 - 3 } & Otros empleos (ej. operario/a) & $32(12,8)$ \\
\cline { 2 - 3 } & Sin información & $76(30,4)$ \\
\hline
\end{tabular}


Tabla 3. Resultados principales: descripción de la información contextual documentada las historias clínicas de 250 adultos familiares de la muestra aleatoria de 153 menores de 18 años con discapacidad documentada, afiliados al seguro de salud de un Hospital Universitario Privado del Área Metropolitana de Buenos Aires. Notas: IC: intervalo de confianza; ICC: intervalo intercuartilo (Percentilo 25 a 75$)$.

${ }^{a}$ UPC: el índice UPC (del inglés, Usual provider of care) sintetiza el número de consultas realizadas al prestador habitual en un periodo determinado, en relación al número total de consultas realizadas a prestadores similares. En este trabajo fue considerado prestador habitual el médico de cabecera más consultado por el paciente durante el período revisado en la historia clínica electrónica ${ }^{8}$.

${ }^{b}$ Contexto global: hace referencia a datos laborales, vínculos familiares, condiciones de vivienda, red de contención, etc.

${ }^{c}$ En este apartado se hace referencia a si estaba consignada la presencia de un familiar con discapacidad en las historias clínicas revisadas.

\begin{tabular}{|c|c|c|c|}
\hline \multicolumn{3}{|l|}{ Desenlace } & Estimación \\
\hline \multicolumn{3}{|c|}{ Índice UPC $^{a}$ de continuidad de los cuidados, mediana (IC $\left.95 \%\right)$} & $0,134(0$ a 0,23$)$ \\
\hline \multicolumn{3}{|c|}{ Episodios de cuidados registrados por el médico de cabecera, mediana (ICC) } & $6(3$ a 12$)$ \\
\hline \multicolumn{3}{|c|}{ Alguna información de su contexto global documentada en la historia clínica electrónica ${ }^{b}, \mathrm{n}$ ( \%) } & $143(57,2)$ \\
\hline \multicolumn{3}{|c|}{ Tiene documentada información sobre el niño o adolescente con discapacidad ${ }^{c}, \mathrm{n}(\%)$} & $117(46,8)$ \\
\hline \multirow{6}{*}{$\begin{array}{l}\text { Sección de historia } \\
\text { clínica donde está } \\
\text { documentada la } \\
\text { información sobre } \\
\text { discapacidad del } \\
\text { familiar, } n(\%)\end{array}$} & \multicolumn{2}{|l|}{ En la lista de problemas } & $18(7,2)$ \\
\hline & \multicolumn{2}{|c|}{ En el registro de una consulta vinculada al problema control de salud } & $76(30,4)$ \\
\hline & \multirow{4}{*}{$\begin{array}{l}\text { En el registro de consultas vinculadas a otros } \\
\text { problemas }\end{array}$} & Ansiedad & $8(3,2)$ \\
\hline & & Crisis familiar & $2(0,8)$ \\
\hline & & Sobrecarga del cuidador & $1(0,4)$ \\
\hline & & Otros & $12(4,8)$ \\
\hline
\end{tabular}

\section{Discusión}

En esta investigación se pudo documentar que, a pesar de que los adultos recibían cuidados longitudinales en un sistema basado en la figura del médico de cabecera, solamente en el $47 \%$ de sus HCE había quedado registrado que en su grupo familiar directo había un menor con discapacidad, mientras que esta información estaba consignada en su lista de problemas sólo en el $7 \%$ de los participantes.

Entre las limitaciones de nuestro diseño vale señalar el hecho de que la falta de documentación en la historia clínica de los participantes no significa que quienes realizan sus cuidados longitudinales no estén al tanto de que en la familia hay un niño con discapacidad. Consideramos tres grandes hipótesis que podrían explicar la omisión de esta relevante información contextual en las historias clínicas revisadas: 1) el médico de cabecera estaba al tanto de dicha información contextual pero había omitido documentarla; 2) el médico de cabecera no estaba al tanto de elementos importantes del contexto de su paciente porque nunca los había indagado de manera específica, probablemente a causa de la gran demanda de problemas de salud de quienes lo consultan; 3) el médico de cabecera había realizado una anamnesis sobre estos temas pero esta información específica había sido omitida en el relato del adulto en cuestión por no haberla considerado relevante, por vergüenza, por temor a ser estigmatizado/a, etc. A su vez, entendemos que si bien con el objetivo de llevar adelante esta investigación seleccionamos a familiares de primer grado como los responsables de los cuidados de las personas con discapacidad, podrían existir otros protagonistas de los cuidados -familiares biológicos u otros convivientes- que no pudimos identificar a través del método utilizado. Por otra parte, no descartamos un potencial subregistro de la información concerniente a algunos vínculos familiares en el padrón de afiliados del asegurador del que fue seleccionada la muestra, por lo que algunos familiares de cada caso índice pudieron no haber sido omitidos al conformar la muestra de participantes de este estudio.

A pesar de todo esto, sea o no conocida la información contextual por el prestador habitual de cuidados de la persona adulta, consideramos muy notorio que en más del $50 \%$ de los participantes de la muestra estudiada no esté documentado en su historia clínica -y menos aún, identificado en su lista problemas- que en grupo familiar de ese adulto hay un menor con discapacidad, en especial dado que se trata de un sistema de salud coordinado por la figura de un médico de cabecera (especialista en clínica médica o medicina de familia). Consideramos probable que la necesidad de atención prioritaria a la agenda de los pacientes, en sus consultas del día a día, resulta una barrera para realizar algunos procesos importantes de cuidado como la conciliación de la medicación, la provisión de consejería o, como en este caso, la anamnesis destinada a conocer y documentar en su historia clínica la información sobre el contexto familiar de las personas a quienes atendemos.

Estos hallazgos son preocupantes ya que actualmente existe amplia recomendación ${ }^{4-7}$ de diseñar modelos de atención para las familias que cuentan con un miembro con discapacidad por sobre el abordaje individual de la persona que la sufre directamente, ya que la discapacidad de un miembro de la familia no solo afecta el desempeño diario de esa persona, sino que modifica los roles y las actividades del grupo familiar y de los cuidadores principales. Por eso, es importante que los médicos de cabecera detectemos entre nuestros pacientes a quienes integran una familia con un miembro con discapacidad, para poder elaborar un plan de cuidados que incluya a todos los participantes, permitiendo así el adecuado cuidado de la persona con dificultades, sin obviar las necesidades del resto de sus miembros.

Si bien quienes diseñamos y ejecutamos esta investigación no estamos sorprendidos de sus resultados, destacamos que este es el primer trabajo que ha documentado en nuestro medio este déficit de documentación en las historias clínicas, y que podría ser interpretado como un indicador indirecto de un proceso de cuidados poco integrado, como lo sugiere el valor del indicador UPC observado en los participantes de la muestra. Queda todavía por delante un largo camino para comprender esta problemática con múltiples aristas, lo que permitirá implementar cambios tendientes a la provisión de cuidados más coordinados de las familias que cuentan con un miembro con discapacidad. 


\section{Conclusiones}

En más de la mitad de la muestra de adultos estudiada no está documentado en su historia clínica electrónica -y menos aún, identificado en su lista de problemas- que en su familia nuclear hay un menor con discapacidad. Estos hallazgos llaman la atención, por tratarse de un sistema de salud que ofrece atención coordinada a través de la figura de un médico de cabecera.
Conflicto de interés de los autores: Los autores no declaran conflictos de interés.

Fuente de financiamiento: El presente estudio contó con el apoyo de la Fundación MF.

Recibido el 25/08/2021 Aceptado el 16/12/2021 y Publicado el 08/01/2022.

\section{Agradecimientos}

A Gabriela Blugerman por la búsqueda y recuperación de los datos administrativos; y a Alberto Antacle y María Julia Yanicelli por sus valiosos aportes durante la revisión de pares que permitieron mejorar el manuscrito.

\section{Referencias}

1. Dirección Nacional de Estadísticas Sociales y de Población. Estudio nacional sobre el perfil de las personas con discapacidad: resultados provisorios 2018. Ciudad Autónoma de Buenos Aires: Instituto Nacional de Estadística y Censos - INDEC; 2018. Available from: https://www.indec.gob.ar/ftp/ cuadros/poblacion/estudio_discapacidad_07_18.pdf.

2. World Health Organization. International Classification of Functioning, Disability and Health: ICF. Diamond Pocket Books (P) Ltd; 2001.

3. Instituto Nacional de Estadística y Censos - I N D E C . Estudio Nacional sobre el Perfil de las Personas con Discapacidad. Resultados definitivos 2018. Ciudad Autónoma de Buenos Aires: Instituto Nacional de Estadística y Censos; 2018. Available from: https://www.indec.gob.ar/ftp/cuadros/ poblacion/estudio_discapacidad_12_18.pdf.

4. Pantano L, Núñez B, Arenaza A. ¿Qué necesitan las familias de personas con discapacidad?: investigación, reflexiones y propuestas. 1st ed. Buenos Aires: Lugar Editorial; 2012. Available from: https://ugareditorial.com.ar/descargas/libros/978-950-892-417-9.pdf.

5. Barrera L. El cuidado del cuidador que afronta enfermedades crónicas. In: and others, editor. Cuidado y práctica de enfermería. vol. 235. Universidad Nacional de Colombia; 2000. p. 233-237. Available from: http://biblio3.url.edu.gt/Publi/Libros/CyPdeEnfermeria/08.pdf.

6. Moreno ME, Náder A, López-De-Mesa C. Relación entre las características de los cuidadores principales de pacientes con discapacidad y la percepción de su estado de salud. Av Enferm. 2004;22(1):27-38. Available from: https://revistas.unal.edu.co/index.php/avenferm/article/view/37935/ 40168.

7. Carrete P. Familias con un miembro con discapacidad. Evid Actual Práct Ambul. 2012;15(1):26-35. Available from: 10.51987/EVIDENCIA.V15I1. 6224.

8. Reid R, Haggerty J, Mckendry R. Defusing the Confusion: Concepts and Measures of Continuity of Healthcare. Ottawa, Canada: Canadian Foundation for Healthcare Improvement/Fondation canadienne pour l'amélioration des services de santé; 2002. 